Commentary

\title{
Response to Professor Olsen's Commentary on 'Random Sampling - Is It Worth It?'
}

\author{
Michael B. Bracken, ${ }^{\mathrm{a}, \mathrm{b}}$ Irva Hertz-Picciotto, ${ }^{\mathrm{c}}$ Michael R. Elliott, ${ }^{\mathrm{d}}$ Donald J. Dudley, ${ }^{\mathrm{g}}$ Stephen Buka, ${ }^{\mathrm{h}}$ \\ James M. Robbins, ${ }^{i}$ Nigel Paneth ${ }^{\mathrm{e}, \mathrm{f}}$ \\ ${ }^{a}$ School of Public Health \\ ${ }^{b}$ School of Medicine, Yale University, New Haven, CT \\ ${ }^{c}$ Department of Public Health Sciences, University of California, Davis, CA \\ ${ }^{d}$ School of Public Health, University of Michigan, Ann Arbor \\ ${ }^{e}$ Department of Epidemiology and Biostatistics \\ ${ }^{f}$ Department of Pediatrics, Michigan State University, East Lansing, MI \\ ${ }^{8}$ Department of Obstetrics and Gynecology, University of Texas Health Sciences Center, San Antonio, TX \\ ${ }^{h}$ Department of Epidemiology, Brown University, Providence, RI \\ ${ }^{i}$ Department of Pediatrics, University of Arkansas, Little Rock, AK
}

Heraclitus was not known as the 'obscure philosopher' without reason. We may not step into the same river twice but it will be a very similar river in the future (salmon - not native to Greece and so perhaps unfamiliar to Heraclitus - have no difficulty returning to the 'same' river 4 years later). Are epidemiologists to abandon all calculations of attributable risk or all attempts at prediction because the actual index population no longer exists? What is the point of randomised trials or, indeed, any research for that matter, if we cannot make assumptions that the population studied is rather similar to the population in which the results of the research will be applied? ${ }^{1}$

The US pregnant population is extremely heterogeneous, and while it may be that true representativeness will never be achieved in the National Children's Study (NCS), even with a valid representative sampling plan, starting out with a population sampling design will surely result in a sample that is much more representative than if a convenience sample is initiated. Moreover, as described in our initial paper, ${ }^{2}$ population-based sampling within the context of a well-defined multistage random sampling design will permit the use of weights that correct for underparticipation, thereby allowing analyses to have external validity. Why even bother with a 'national' children's study if not to have the capacity to draw conclusions applicable beyond the enrolees who are de facto distinguished from non-participants by their interest and

Correspondence: Michael B. Bracken, Yale School of Medicine, School of Public Health, Center for Perinatal, Pediatric and Environmental Epidemiology, New Haven, CT 06510, USA. E-mail: michael.bracken@yale.edu commitment to research as well as sociodemographic and other characteristics? In addition to being able to accurately estimate prevalence and attributable risk, having a probability sample helps ensure that associations are more validly estimated, for example, avoiding bias due to inadvertently over- or under-selecting high or low exposed children differentially by disease status.

Even with an NCS total sample of 100 000, stratification into groups of particular interest is likely to substantially reduce the power to test hypotheses in some subpopulations at particular risk: the underserved, those without any prenatal care and minority populations. Convenience sampling is especially likely to underrepresent these groups. However, a population-based sample, which enhances the intensity of recruitment strategies in these populations, will improve the chances of more balanced final sample sizes in these groups. Indeed, 'health disparities' is one area of study mandated by the Children's Health Act of 2000 that led to the NCS. Populations deprived of health care access, health information, healthy diets and other amenities enjoyed by more privileged sectors of the population must be adequately represented to undertake valid research on disparities. Options to obtain 'supplemental' samples of underserved populations currently being considered by the NCS leadership are entirely inadequate. They would be obtained from different populations and using procedures incomparable to the majority groups enrolled through the primary recruitment strategy.

There is no evidence that recruiting a probability sample is any more expensive in money or time than 
non-representative strategies. Posing internal and external validity as being at odds with each other is a curious twist: in what way does population-based sampling for a large cohort detract from internal validity? For that matter, how is probability sampling less ethical than convenience sampling? On the other hand, embarking on a study that is destined to be unable to answer some of the major questions concerning pregnancy and disease in underserved populations strikes us as being highly irresponsible.

If high impact rare diseases (such as autism) and complex childhood disorders (for example, Type 1 and increasingly Type 2 diabetes) are to be studied within the NCS, it cannot be left entirely to chance that sufficient cases are sampled. Similarly, rare exposures can only be studied with some degree of precision in a sample that captures the broad range of environmental agents to which US women, and their fetuses and children, are exposed. This includes as yet unknown and unanticipated exposures which may become of future interest.

The Danes are fortunate in having several linked complete population registries which Professor Olsen and many colleagues have used to great advantage in studying reproductive and perinatal health. Fortunately for them, sampling was not necessary. Among the many benefits of reasonably complete national registries is the capacity to contrast participants from non-participants and to adjust for these in statistical analyses and to recognise any resulting limitations to external validity from the enrolled subjects. But national registries of this type in the US are a pipe dream and the closest that will be achieved are population-based samples. In the absence of probability sampling, the scientific utility of the NCS will be a fraction of its full potential. It is no coincidence that each of the many advisory groups and panels of sampling experts to have considered the NCS study design has without exception endorsed probability sampling, ${ }^{3,4}$ including a major review by the Institute of Medicine. ${ }^{5}$

\section{References}

1 Olsen J. Random sampling - is it worth it? Commentary to Belanger et al. Implementing provider-based sampling for the National Children's Study: opportunities and challenges. Paediatric and Perinatal Epidemiology. In press.

2 Belanger K, Buka S, Cherry DC, Dudley DJ, Elliott MR, Hale $\mathrm{DE}$, et al. Implementing provider-based sampling for the National Children's Study: opportunities and challenges. Paediatric and Perinatal Epidemiology 2012 Sept 27. Epub ahead of print.

3 Kaiser J. Advisers say National Children's Study should represent U.S. population. Science Insider, April 25th 2012.

4 Wadman M. Scientists urge broad sampling for US children's study. Nature News Blog, April 24th 2012.

5 National Research Council, Institute of Medicine. The National Children's Study Research Plan: A Review. Washington, DC: The National Academies Press, 2008. 